\title{
Spinal Inflammation
}

In part because of the epidemic of acquired immunodeficiency syndrome (AIDS), the frequency of central nervous system (CNS) infections has been on the rise (Post et al., 1986; Provenzale and Jinkins, 1997; Thurnher et al., 1997). The use of diagnostic imaging in the evaluation of infections involving the CNS has become an essential component of patient care. Patients with inflammatory disease of the CNS usually present with nonspecific signs and symptoms that often require a more detailed investigation. Early delineation of the location, extent, and type of infection may be an important determinant of outcome. While the modern imaging of spinal inflammatory conditions can be carried out using various methods ranging from conventional radiography through the newer computed imaging techniques, MRI has proven to be the most sensitive, if not specific, imaging technique. This unit will outline the various techniques realizing this potential (Modic et al., 1985; Chang et al., 1989; Sharif et al., 1990; Sklar et al., 1993; Jinkins et al., 1995; Reddy et al., 1995).

\section{CONVENTIONAL AND FAST SPIN ECHO}

Improvements in technology have made MR the investigation of choice in the diagnostic evaluation of spinal infections. MR provides anatomic localization and characterization of spinal infections that is far superior to any other imaging technique. MR imaging has the advantage of multiplanar direct visualization of the spinal cord, subarachnoid space, extradural soft tissues, and spinal column noninvasively without necessitating the intrathecal injection of a contrast agent. In addition, pathologic changes in the spine are seen earlier on MR than on any other imaging modality. Another advantage of MR is its easy sequential repeatability and reproducibility in patients undergoing treatment for spinal infection.

The following sequences comprise the preferred protocol for high field MR machines. In addition, in all cases intravenous (i.v.) contrast enhanced protocols are mandatory to evaluate the integrity of the blood-nerve and blood cord barrier in certain cases of suspected CNS infection, or in cases of epidural and/or spinal column/paraspinal infection (e.g., spondyloclodiscitis and perispinal abscess/phlegmon).

Table A8.4.1 lists the hardware necessary to perform the procedure, along with appropriate parameters. The available gradient strength will depend on the scanner, and the echo times given in other tables will be varied accordingly (i.e., the smaller the gradient strength, the longer the echo time for a particular scan).

This entire protocol should take 40 to $60 \mathrm{~min}$ to complete.

\begin{tabular}{ll}
$\begin{array}{l}\text { Table A8.4.1 } \\
\text { Inflammation }\end{array}$ & Equipment Parameters for Spine Imaging in Cases of \\
\hline Coil type & $\begin{array}{l}\text { Cervical, thoracic, lumbar: phase array } \\
\text { surface coil (or other depending upon } \\
\text { machine compatibility and availability) } \\
\end{array}$ \\
$\begin{array}{l}\text { Gradient coil strength } \\
\text { Flow compensation }\end{array}$ & $\begin{array}{l}\text { Optional (any level, if available) } \\
\text { Peripheral gating }\end{array}$ \\
$\begin{array}{l}\text { Respiratory gating } \\
\text { Use of contrast agents }\end{array}$ & Optional (thoracic spine only) \\
\hline
\end{tabular}

BASIC PROTOCOL

Extradural Spine

A8.4.1

Supplement 1 
NOTE: Be sure that technicians and nurses have immediate access to any emergency equipment that may be relevant to a given study, or that may be needed for a particular patient, such as crash carts or oxygen.

\section{Materials}

Normal saline $(0.9 \% \mathrm{NaCl})$, sterile

Gadolinium-based MR contrast agent (e.g., Magnevist, Omniscan, or Prohance)

\section{Set up patient and equipment}

1. Interview (screen) the patient to ensure that he or she has no contraindications such as cardiac pacemakers or other implants containing ferromagnetic materials. Also be sure to find out if the patient has any health conditions that may require the presence of special emergency equipment during the scanning procedure, or necessitate any other precautions.

Generally, standard screening forms are used for all patients scanned in a magnetic resonance system.

The presence of any ferromagnetic materials may be a health hazard to the patient when he or she is inside the magnet, and will also affect the imaging. If in doubt as to the exact composition of the items, it is best to exclude patients with any implants; see Shellock (1996) for discussion of what implants may be safely scanned using magnetic resonance.

Patients may be accompanied into the magnet room by a friend or family member, who can sit in the room during the scan and comfort the patient as needed. This companion must be screened as well to ensure the absence of loose metal objects on the body or clothing.

2. If the procedure is a research protocol, have the patient sign any necessary consent form.

3. Have the patient remove all jewelry and change into a gown to eliminate any metal that might be found in clothing.

4. Inform the patient about what will occur during the procedure, what he or she will experience while in the magnet, and how to behave, including the following.

a. If earphones or headphones are used to protect the ears from the loud sounds produced by the gradients, the patient will be asked to wear these, but will be able to communicate with you at any time during the imaging.

b. The patient will be given a safety squeeze-bulb or similar equipment to request assistance at any time (demonstrate how this works).

c. For good results, the patient should not talk, and should avoid or minimize swallowing or other movement, during each scan-i.e., as long as the banging sounds continue. Between scans, talking and swallowing are allowed in most cases, but should be avoided when comparative positional studies are being performed; the patient will be informed when this is the case.

d. Nevertheless, the patient may call out at any time if he or she feels it necessary.

5. Have the patient mount onto the table. Either before or right after the patient lies down, set up any triggering devices or other monitoring equipment that is to be used.

6. Center the coil over the region where the key information is desired.

Make sure that the body is constrained to prevent motion, especially if high-resolution scans are to be run.

7. If needed, place a pillow or other support under the knees to make the patient more comfortable. 
Table A8.4.2 Primary Clinical Imaging Parameters for Sequence 1 (Pilot Scan)

\begin{tabular}{ll}
\hline Patient position & Supine \\
Scan type & Gradient echo \\
Imaging plane (orientation) & Transverse, sagittal, coronal \\
Central slice or volume center & Laser light centered at: \\
& Cervical spine: thyroid cartilage \\
& Thoracic spine: nipple line \\
& Lumbar spine: iliac crests \\
Echo time $\left(T_{\mathrm{E}}\right)$ & As short as possible \\
Repeat time $\left(T_{\mathrm{R}}\right)$ & As short as possible \\
Flip angle $(\mathrm{FA})$ & $15^{\circ}$ \\
Fields of view $(\mathrm{FOV}, \mathrm{FOV}$ & \\
& Cervical: $240 \mathrm{~mm}, 240 \mathrm{~mm}$ \\
& Thoracic: $320 \mathrm{~mm}, 320 \mathrm{~mm}$ \\
Resolution $(\Delta x, \Delta y)$ & Lumbosacral: $280 \mathrm{~mm}, 280 \mathrm{~mm}$ \\
& Cervical: $0.94 \mathrm{~mm}, 0.94 \mathrm{~mm}$ \\
Number of data points collected $\left(N_{\mathrm{x}}, N_{\mathrm{y}}\right)$ & Thoracic: $1.25 \mathrm{~mm}, 1.25 \mathrm{~mm}$ \\
Display matrix $\left(D_{\mathrm{x}}, D_{\mathrm{y}}\right)$ & Lumbrosacral: $1.09 \mathrm{~mm}, 1.09 \mathrm{~mm}$ \\
Slice thickness $(\Delta z)$ & 256,256 \\
Number of slices & 5 mm \\
Slice gap & 3 \\
Number of acquisitions $\left(N_{\mathrm{acq}}\right)$ & Not applicable \\
Scan time & 1 \\
\hline
\end{tabular}

8. Use the centering light to position the patient (cervical spine: thyroid cartilage; thoracic spine: nipple line; lumbar spine: iliac crests) and put him or her into the center of the magnet.

Once this step has been performed, so long as the patient does not move on the table, the table itself can be moved and then replaced in the same position as before without jeopardizing the positioning of one scan relative to another.

9. If the patient is unable to hold still, provide an appropriate sedative.

\section{Sequence 1: Rapid positioning pilot}

10. To validate the patient's position, run the system's pilot (or scout) scan (sequence 1) to ensure correct location of the neck in three dimensions, using the imaging sequence given in Table A8.4.2 or similar parameters.

This sequence usually consists of three orthogonal planes to allow subsequent localization. The images are often also used later to determine where to place the saturation pulses and to set up total coverage of the volume of interest.

\section{Sequence 2: Sagittal $T_{1}$-weighted conventional spin echo}

11. Set the imaging parameters as shown in Table A8.4.3.

12. Use the pilot image to locate the spine in three dimensions to ensure coverage of the region of interest (e.g., cervical, thoracic, lumbosacral spine).

13. Let the patient know you are ready and begin the scan.

Extradural Spine

A8.4.3

Supplement 1 
Table A8.4.3 Primary Clinical Imaging Parameters for Sequence 2 ( $T_{1}$-Weighted Image)

\begin{tabular}{|c|c|}
\hline Patient position & Supine \\
\hline Scan type & Conventional spin echo \\
\hline Imaging plane (orientation) & Sagittal \\
\hline \multirow[t]{4}{*}{ Central slice or volume center } & Centered on: \\
\hline & Cervical: 3rd cervical vertebra \\
\hline & Thoracic: 6 th thoracic vertebra \\
\hline & Lumbar: third lumbar vertebra \\
\hline Echo time $\left(T_{\mathrm{E}}\right)$ & $10 \mathrm{msec}$ \\
\hline Repeat time $\left(T_{\mathrm{R}}\right)$ & $500 \mathrm{msec}$ \\
\hline Flip angle (FA) & $90^{\circ}$ \\
\hline \multirow[t]{4}{*}{ Fields of view $\left(\mathrm{FOV}_{\mathrm{x}}, \mathrm{FOV}_{\mathrm{y}}\right)$} & Cervical: $240 \mathrm{~mm}, 240 \mathrm{~mm}$ \\
\hline & Thoracic: $320 \mathrm{~mm}, 320 \mathrm{~mm}$ \\
\hline & Lumbosacral: 280 mm, 280 mm (may \\
\hline & $\begin{array}{l}\text { use rectangular field of view, e.g., half } \\
\text { or three-quarter field, if available, or } \\
\text { tailor to region of interest) }\end{array}$ \\
\hline \multirow[t]{3}{*}{ Resolution $(\Delta x, \Delta y)$} & Cervical: $0.94 \mathrm{~mm}, 0.94 \mathrm{~mm}$ \\
\hline & Thoracic: $1.25 \mathrm{~mm}, 1.25 \mathrm{~mm}$ \\
\hline & Lumbosacral: $1.09 \mathrm{~mm}, 1.09 \mathrm{~mm}$ \\
\hline Number of data points collected $\left(N_{\mathrm{x}}, N_{\mathrm{y}}\right)$ & 256,256 \\
\hline Display matrix $\left(D_{\mathrm{x}}, D_{\mathrm{y}}\right)$ & 256,256 \\
\hline \multirow[t]{3}{*}{ Slice thickness $(\Delta z)$} & Cervical: $3 \mathrm{~mm}$ \\
\hline & Thoracic: $3 \mathrm{~mm}$ \\
\hline & Lumbar: $1 \mathrm{~mm}$ \\
\hline Number of slices & $\begin{array}{l}\text { As many as needed to cover the region } \\
\text { of interest }\end{array}$ \\
\hline \multirow[t]{3}{*}{ Slice gap } & Cervical: $0.5 \mathrm{~mm}$ \\
\hline & Thoracic: $1 \mathrm{~mm}$ \\
\hline & Lumbar: $1 \mathrm{~mm}$ \\
\hline Number of acquisitions $\left(N_{\text {acq }}\right)$ & 2 \\
\hline Flow compensation & Yes (if available) \\
\hline Saturation pulses & $\begin{array}{l}\text { Yes; anterior cervical/thoracic/lumbar } \\
\text { slab to saturate larynx/vessels }\end{array}$ \\
\hline Slice series & $\begin{array}{l}\text { Left to right or the reverse depending } \\
\text { on preference }\end{array}$ \\
\hline Scan time & $\sim 4 \min$ \\
\hline
\end{tabular}

Sequence 3: Sagittal $T_{2}$-weighted fast spin echo, fat suppressed

14. Review the pilot scans and ensure that the saturation pulse is correctly placed anterior to above the slab of interest.

15. Run sequence 3 according to Table A8.4.4.

\section{Sequence 4: Transverse $T_{1}$-weighted conventional spin echo}

16. Using the midline sagittal $T_{1}$-weighted image acquired in Sequence 2, set the transverse acquisition parameters as follows:

a. Cervical spine: stacked images from $\mathrm{C} 1$ through $\mathrm{C} 1-\mathrm{T} 1$

b. Thoracic spine: stacked images through levels of interest 
Table A8.4.4 Primary Clinical Imaging Parameters for Sequence 3 ( $T_{2}$-Weighted Image, FSE) ${ }^{a}$

\begin{tabular}{ll}
\hline Patient position & Supine \\
Scan type & Fast spin echo \\
Imaging plane (orientation) & Sagittal \\
Central slice or volume center & Centered on area of interest (as in \\
& Sequence 2, Table A8.4.3) \\
Echo time $\left(T_{\mathrm{E}}\right)$ & $100 \mathrm{msec}$ \\
Echo train length $(\mathrm{ETL})$ & 8 \\
Repeat time $\left(T_{\mathrm{R}}\right)$ & $4000 \mathrm{msec}$ \\
Flip angle $(\mathrm{FA})$ & $90^{\circ}$ \\
Fields of view $\left(\mathrm{FOV}, \mathrm{FOV} \mathrm{y}_{\mathrm{y}}\right)$ & As in Sequence 2, Table A8.4.3 \\
Resolution $(\Delta x, \Delta y)$ & Cervical: $0.47 \mathrm{~mm}, 0.47 \mathrm{~mm}$ \\
& Thoracic: $0.63 \mathrm{~mm}, 0.63 \mathrm{~mm}$ \\
& Lumbosacral: $0.55 \mathrm{~mm}, 0.55 \mathrm{~mm}$ \\
Number of data points collected $\left(N_{\mathrm{x}}, N_{\mathrm{y}}\right)$ & 512,512 \\
Display matrix $\left(D_{\mathrm{x}}, D_{\mathrm{y}}\right)$ & 512,512 \\
Slice thickness $(\Delta z)$ & Cervical: $3 \mathrm{~mm}$ \\
& Thoracic: $3 \mathrm{~mm}$ \\
Number of slices & Lumbar: $1 \mathrm{~mm}$ \\
Slice gap & Varies with spinal level \\
& Cervical: $0.5 \mathrm{~mm}$ \\
Number of acquisitions $\left(N_{\mathrm{acq}}\right)$ & Thoracic: $1 \mathrm{~mm}$ \\
Flow compensation & Lumbar: $1 \mathrm{~mm}$ \\
Saturation pulses & 1 \\
Fat suppression & Yes (if available) \\
Slice series & Yes; anterior cervical/thoracic/lumbar \\
& slabs to saturate larynx/vessels/heart \\
& Yes: chemical saturation or STIR \\
(short tau inversion recovery) & Left to right or the reverse depending \\
on preference & $\sim 4$ min \\
\hline
\end{tabular}

${ }^{a}$ FSE: fast spin echo.

c. Lumbosacral spine: 5 slices each, angled to the plane of the intervertebral disc at L3-4, L4-5, and L5-S1; one or more slices each, angled to the intervertebral disc at L1-2 and L2-3.

17. Supplement additional slices according to visible disease present or to clinical query.

18. Run sequence 4 according to Table A8.4.5.

\section{Sequence 5: Transverse $T_{1}$-weighted fast spin echo}

19. Using the midline $T_{1}$-weighted image acquired in sequence 2 , repeat the setup as in Table A8.4.6.

20. Run sequence 5 according to Table A8.4.6.

\section{Sequences 6 and 7: Sagittal (Sequence 6) and transverse (Sequence 7) i.v. contrast enhanced $T_{1}$-weighted spin echo}

21. Remove patient from the magnet. Using the same equipment, set up equipment and patient as in steps 5 to 9 . 
Table A8.4.5 Primary Clinical Imaging Parameters for Sequence 4 ( $T_{1}$-Weighted Image)

\begin{tabular}{|c|c|}
\hline Patient position & Supine \\
\hline Scan type & Conventional spin echo \\
\hline Imaging plane (orientation) & Transverse \\
\hline Central slice or volume center & $\begin{array}{l}\text { Centered on the area of interest (as in } \\
\text { Sequence 2, Table A8.4.3) }\end{array}$ \\
\hline Echo time $\left(T_{\mathrm{E}}\right)$ & $10 \mathrm{msec}$ \\
\hline Repeat time $\left(T_{\mathrm{R}}\right)$ & $500 \mathrm{msec}$ \\
\hline Flip angle (FA) & $90^{\circ}$ \\
\hline Fields of view $\left(\mathrm{FOV}_{\mathrm{x}}, \mathrm{FOV}_{\mathrm{y}}\right)$ & As in Sequence 2, Table A8.4.3 \\
\hline \multirow[t]{3}{*}{ Resolution $(\Delta x, \Delta y)$} & Cervical: $0.94 \mathrm{~mm}, 0.94 \mathrm{~mm}$ \\
\hline & Thoracic: $1.25 \mathrm{~mm}, 1.25 \mathrm{~mm}$ \\
\hline & Lumbosacral: $1.09 \mathrm{~mm}, 1.09 \mathrm{~mm}$ \\
\hline Number of data points collected $\left(N_{\mathrm{x}}, N_{\mathrm{y}}\right)$ & 256,256 \\
\hline Display matrix $\left(D_{\mathrm{x}}, D_{\mathrm{y}}\right)$ & 256,256 \\
\hline \multirow[t]{3}{*}{ Slice thickness $(\Delta z)$} & Cervical: $3 \mathrm{~mm}$ \\
\hline & Thoracic: $3-8 \mathrm{~mm}$ \\
\hline & Lumbar: $4 \mathrm{~mm}$ \\
\hline Number of slices & Varies with spinal level \\
\hline \multirow[t]{3}{*}{ Slice gap } & Cervical: $1 \mathrm{~mm}$ \\
\hline & Thoracic: $1-2 \mathrm{~mm}$ \\
\hline & Lumbar: $1 \mathrm{~mm}$ \\
\hline Number of acquisitions $\left(N_{\text {acq }}\right)$ & 2 \\
\hline Slice locations & See text (Basic Protocol, step 16) \\
\hline Flow compensation & Yes (if available) \\
\hline Scan time & $\sim 4 \mathrm{~min}$ \\
\hline
\end{tabular}

22. Establish an i.v. line from which the contrast agent can be injected, and attach this line securely to the patient so that movement into or out of the magnet will not pull at the patient's arm.

It is preferable to insert the line prior to imaging and to leave the patient in the magnet, with no intervening motion, between the scans that are run before contrast agent injection and those run after injection.

\section{Run pilot scan}

23. Run a rapid three-plane positioning pilot scan (see sequence 1).

\section{Scan sequences}

24. Leaving the patient in the magnet, inject the contrast agent, flush the i.v. line with 10 cc saline, and then immediately run sagittal (sequence 6) and transverse (sequence 7) $T_{1}$-weighted image sequences (see sequences 2 and 4).

A dose of $0.1 \mathrm{mmol} / \mathrm{kg}$ of contrast agent is usually given i.v.

This is performed to demonstrate solid or cavitary infectious disease (Post et al., 1990).

Fat suppression techniques may be very useful in order to suppress osseous vertebral marrow fat and perispinal soft tissue fat, thereby clearly distinguishing enhancement from normal fatty tissue. Fat saturation or STIR (short tau inversion recovery) techniques may be used to suppress fat. 
Table A8.4.6 Primary Clinical Imaging Parameters for Sequence 5 ( $T_{2}$-Weighted Image, FSE) ${ }^{a}$

\begin{tabular}{ll}
\hline Patient position & Supine \\
Scan type & Fast spin echo \\
Imaging plane (orientation) & Transverse \\
Central slice or volume center & Centered on the region of interest (as \\
& in Sequence 2, Table A8.4.3) \\
Echo time $\left(T_{\mathrm{E}}\right)$ & $100 \mathrm{msec}$ \\
Echo train length $(\mathrm{ETL})$ & 6 \\
Repeat time $\left(T_{\mathrm{R}}\right)$ & $4000 \mathrm{msec}$ \\
Flip angle $(\mathrm{FA})$ & $90^{\circ}$ \\
Fields of view $\left(\mathrm{FOV}_{\mathrm{x}}, \mathrm{FOV}_{\mathrm{y}}\right)$ & As in Sequence 2, Table A8.4.3 \\
Resolution $(\Delta x, \Delta y)$ & Cervical: $0.94 \mathrm{~mm}, 0.94 \mathrm{~mm}$ \\
& Thoracic: $1.25 \mathrm{~mm}, 1.25 \mathrm{~mm}$ \\
& Lumbosacral: $1.09 \mathrm{~mm}, 1.09 \mathrm{~mm}$ \\
Number of data points collected $\left(N_{\mathrm{x}}, N_{\mathrm{y}}\right)$ & 256,256 \\
Display matrix $\left(D_{\mathrm{x}}, D_{\mathrm{y}}\right)$ & 256,256 \\
Slice thickness $(\Delta z)$ & Cervical: $3 \mathrm{~mm}$ \\
& Thoracic: $3-8 \mathrm{~mm}$ \\
Number of slices & Lumbar: $4 \mathrm{~mm}$ \\
Slice gap & Varies with spinal level \\
& Cervical: $1 \mathrm{~mm}$ \\
Number of acquisitions $\left(N_{\mathrm{acq}}\right)$ & Thoracic: $1-2 \mathrm{~mm}$ \\
Slice locations & Lumbar 1 mm \\
Flow compensation & 2 \\
Saturation pulses & See text (Basic Protocol, step 16) \\
Scan time & Yes (if available) \\
\hline
\end{tabular}

${ }^{a}$ FSE: fast spin echo.

\section{CORONAL CONVENTIONAL SPIN ECHO-ENHANCED}

In some instances, a coronal acquisition may be helpful to analyse the perispinal tissues for abscess formation.

\section{Run pilot scan}

1. Run a rapid three-plane positioning pilot scan (see Basic Protocol, sequence 1).

\section{Sequence 8: Contrast enhanced coronal $T_{1}$-weighted sequence}

2. Leaving patient in the magnet, inject the contrast agent, flush i.v. line with $10 \mathrm{ml}$ saline, and then immediately run sagittal (sequence 6) and transverse (sequence 7) $T_{1}$-weighted image sequences (see Basic Protocol, sequences 2 and 4).

A dose of $0.1 \mathrm{mmol} / \mathrm{kg}$ of contrast agent is usually given.

Fat suppression techniques may be very useful in order to suppress osseous vertebral marrow fat and perispinal soft tissue fat, thereby clearly distinguishing enhancement from normal fatty tissue.

3. Run the sequence according to Table A8.4.7.

As stated above, fat suppression may be useful (see sequences 6 and 7).

Extradural Spine

A8.4.7

Supplement 1 
Table A8.4.7 Primary Clinical Imaging Parameters for Sequence 8 ( $T_{1}$-Weighted Image)

\begin{tabular}{ll}
\hline Patient position & Supine \\
Scan type & Conventional spin echo \\
Imaging plane (orientation) & Coronal \\
Central slice or volume center & $\begin{array}{l}\text { Centered on the area of interest (as in } \\
\text { sequence 2, Table A8.4.3) } \\
\end{array}$ \\
Echo time $\left(T_{\mathrm{E}}\right)$ & $500 \mathrm{msec}$ \\
Repeat time $\left(T_{\mathrm{R}}\right)$ & $90^{\circ}$ \\
Flip angle $(\mathrm{FA})$ & As in Sequence 2, Table A8.4.3 \\
Fields of view $\left(\mathrm{FOV}_{\mathrm{x}}, \mathrm{FOV}_{\mathrm{y}}\right)$ & Cervical: $0.94 \mathrm{~mm}, 0.94 \mathrm{~mm}$ \\
Resolution $(\Delta x, \Delta y)$ & Thoracic: $1.25 \mathrm{~mm}, 1.25 \mathrm{~mm}$ \\
& Lumbosacral: $1.09 \mathrm{~mm}, 1.09 \mathrm{~mm}$ \\
& 256,256 \\
Number of data points collected $\left(N_{\mathrm{x}}, N_{\mathrm{y}}\right)$ & 256,256 \\
Display matrix $\left(D_{\mathrm{x}}, D_{\mathrm{y}}\right)$ & $3-4 \mathrm{~mm}$ \\
Slice thickness $(\Delta z)$ & Varies with spinal level \\
Number of slices & $1-2 \mathrm{~mm}$ \\
Slice gap & 2 \\
Number of acquisitions $\left(N_{\mathrm{acq}}\right)$ & From front to back of spinal column \\
Slice locations & No \\
Saturation pulses & Yes: fat suppression or STIR (short tau \\
Fat suppression & inversion recovery) \\
Scan time & $\sim 4$ min
\end{tabular}

\section{COMMENTARY}

\section{Background Information}

The most common symptoms of spinal infection include malaise, back pain, and focal tenderness. Spinal infections may present with signs that vary in degree of severity from a mild fever to a life-threatening septicemia and paraplegia. Compromised hosts such as diabetics, intravenous drug abusers, and chronically ill patients are particularly susceptible to spinal infections. In patients who are not abusing intravenous medications, the most common route of spread to the spine is still a hematogenous one, originating from the genitourinary tract, gastrointestinary tract, gastrointestinal tract, skin, or respiratory tract. Infections of the spine also can spread via direct extension from infections involving adjacent paraspinal tissues, or by inoculation occurring from penetrating trauma, therapeutic procedure, or diagnostic studies. The usual pathologic manifestations are the disk space infection, osteomyelitis of the vertebral body (or bodies), epidural phlegmon (solid soft tissue inflammation), epidural abscess, meningitis, paraspinal phlegmon/abscess, and intramedullary spinal cord myelitis, granuloma, or abscess. Infection within the vertebral body usually starts in the anterior (ventral) subchondral region. It can spread to the adjacent disk and/or directly to adjacent vertebrae, sparing the disk by tracking beneath the longitudinal ligaments in the potential subligamentous space. Alternatively, the infection can be disseminated directly to the leptomeninges or the parenchyma of the spinal cord. This may result in arachnoiditis, myelitis, or meningeal/parenchymal granuloma/abscess formation.

It has been reported that MR has a sensitivity of $96 \%$, a specificity of $92 \%$, and an accuracy of $94 \%$ in the diagnosis of vertebral osteomyelitis. This is roughly equivalent to the combined results of technetium radionuclide bone scans and gallium radionuclide studies.

MR is also the most sensitive imaging modality for the detection of diskitis (Modic et al., 1985). Sagittal sections are especially useful for demonstrating infection of the disk space and adjacent vertebral body. The transverse sections are excellent for assessing paravertebral soft tissue extension. The infected disk 

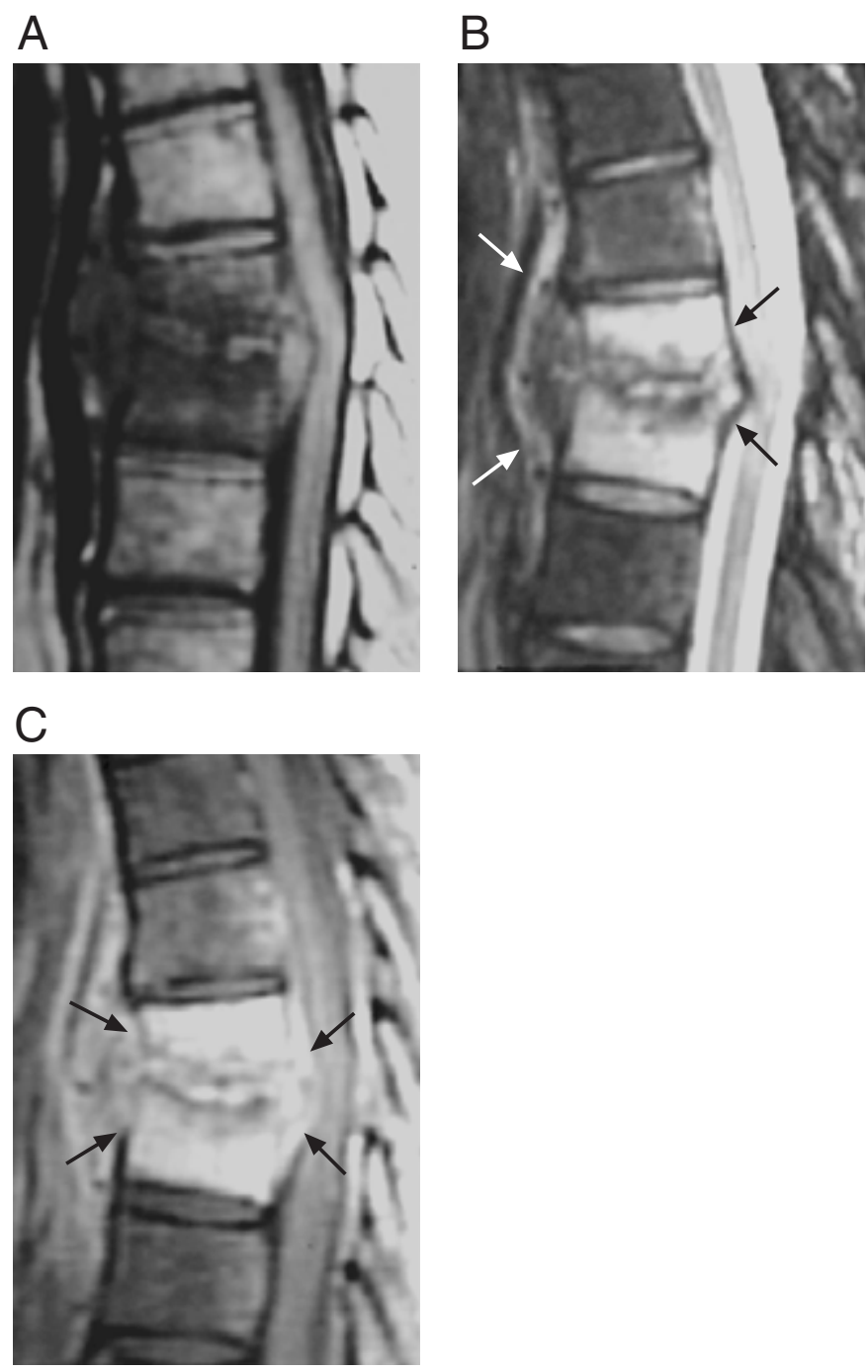

Figure A8.4.1 Thoracic spondylodiscitis (Staphylococcus aureus). (A) Sagittal $T_{1}$-weighted ( $T_{\mathrm{R}}$ $=500 \mathrm{msec}, T_{\mathrm{E}}=10 \mathrm{msec}$ ) image shows poor definition of the intervertebral disc at T5-T6 and an anterior and epidural spinal mass. Also the vertebral body marrow of T5 and T6 is hypointense. (B) Sagittal $T_{2}$-weighted ( $T_{\mathrm{R}}=4000 \mathrm{msec}, T_{\mathrm{E}}=100 \mathrm{msec}$ ) MR shows abnormal hyperintensity in the marrow of T5 and T6 with early segmental collapse of the spinal column. Anterior epidural and prevertebral mass for motion (arrows) is also noted. (C) Intravenous gadolinium enhanced $T_{1}$ weighted $\left(T_{\mathrm{R}}=500 \mathrm{msec}, T_{\mathrm{E}}=10 \mathrm{msec}\right)$ image shows abnormal enhancement of the T5 and T6 vertebral body marrow, as well as the enhancing prespinal and anterior epidural inflammatory spinal mass (arrows).

space and portions of the vertebral bodies adjacent to the disk are relatively hypointense on $T_{1}$-weighted imaging following gadolinium administration, including thin or thick enhancement of the periphery of the disk or diffuse enhancement of the majority of the disk space. A loss of disk height and paravertebral soft tissue alterations are also typically observed. With increasing involvement of the adjacent vertebral bodies in the infectious process, the clear distinction between intervertebral disk and vertebral body becomes increasingly difficult on MR imaging. The associated increase in water content of the disk and adjacent bone is reflected in the signal changes on $T_{1^{-}}$and $T_{2}$-weighted imaging. These MR signal alterations usually precede lesions visible on conventional radiographs of the spine. Malignant lesions can usually be differentiated from infection by the sparing of disk spaces in the former
Extradural Spine

A8.4.9 
condition. However, in the early stages of osteomyelitis, where only the vertebral body is involved, this differentiation becomes difficult.

Gadolinium $(\mathrm{Gd})$ is valuable in cases of suspected spinal infection because it demonstrates the focus of spinal involvement, directs needle biopsy if necessary, and provides a sensitive means of following the resolving infection on medical therapy (Post et al., 1990). In one study, although the diagnosis of discitis and osteomyelitis could not be made in all patients on the basis of unenhanced MR alone, enhancement with intravenous Gd resulted in the correct diagnosis in all cases.

Gd-enhanced studies also provide good demarcation of the epidural or paraspinous soft tissue spread of spinal infection. The majority of epidural inflammatory processes homogeneously enhance, indicating that they are predominantly phlegmonous (Fig. A8.4.1). On the other hand, extradural abscesses have a sharp margin of enhancement surrounding the central nonenhancing fluid pus.

Gd-enhanced MR is the investigation of choice in cases of intramedullary infection of the spinal cord. The enhanced MR may show a ring- or solid-enhancing lesion within the substance of an enlarged cord. There is invariably a surrounding area of nonenhancing cord edema. In acute/subacute infectious arachnoiditis, Gd-enhanced MR shows matting of nerve roots and diffuse leptomeningeal and nerve root enhancement.

\section{Critical Parameters and Troubleshooting}

Cerebrospinal fluid (CSF) flow, as well as cardiac, laryngeal, body wall, and other sources can produce artifacts that can on occasion significantly degrade the images. Proper spatial saturation pulses, cardiac/respiratory gating, and flow compensation gradients can reduce these artifacts significantly. In spite of this, in some instances these artifacts may be difficult or impossible to overcome in any given case.

\section{Anticipated Results}

The goal of studying the spine in cases of clinically suspected inflammation involving the spinal column and peri-intraspinal tissues is to confirm this diagnosis, exclude other etiologies of disease if possible, and to determine the focus and extent of the inflammatory disease. On occasion spinal inflammation can be confused with other types of pathology such as neoplasia. Although such specificity is a relative limitation of MRI, this is easily overcome in most cases by imaging directed needle biopsy or aspiration.

\section{Literature Cited}

Chang, K.H., Han, M.H., Choi, Y.W, et al. 1989. Tuberculous arachnoiditis of the spine: Findings on myelography, CT, and MR imaging. Am.J. Neuroradiol. 10:1255-1262.

Jinkins, J.R., Gupta, R., Chang, K.H., and Rodriguez-Carbajal, J. 1995. MR imaging of central nervous system tuberculosis. Radiol. Clin. North Am. 33:771-786.

Modic, M.T., Feiglin, D.H., Piraino, D.W., et al. 1985. Vertebral osteomyelitis: Assessment using MR. Radiology 157:157-166.

Post, M.J.D., Sheldon, J.J., Hensley, G.T., et al. 1986. Central nervous system disease in acquired immunodeficiency syndrome: Prospective correlation using CT, MR imaging, and pathologic studies. Radiology 158:141-148.

Post, M.J.D., Sze, G., Quencer, R.M., et al. 1990. Gadolinium enhanced MR in spinal infection. $J$. Comput. Assist. Tomogr. 15:721-729.

Provenzale, J.M. and Jinkins, J.R. 1997. Brain and spine imaging findings in AIDS patients. Radiol. Clin. North Am. 35:1127-1166.

Reddy, S., Leite, C.C., and Jinkins, J.R. 1995. Imaging of infectious disease of the spine. In Spine: State of the Art Reviews, Vol. 9 (R.R. Lee, ed.) pp. 119-140. Hanley \& Belfus, Philadelphia.

Sharif, H.S., Clark, D.C., Aabed, M.Y., et al. 1990. Granulomatous spinal infections: MR imaging. Radiology 177:101-107.

Shellock, F.G. 1996. Pocket Guide to MR Procedures and Metallic Objects. Lippincott-Raven, Philadelphia.

Sklar, E.M., Post, M.J.D., and Lebwohl, N.H. 1993. Imaging of infection of the lumbosacral spine. Neuroimag. Clin. North Am. 3:577-590.

Thurnher, M.M., Jinkins, J.R., and Post, M.J.D. 1997. Diagnostic imaging of infections and neoplasms affecting the spine in patients with AIDS. Neuroimag. Clin. North Am. 7:341-357.

Contributed by J. Randy Jinkins and David D. Stark

Downstate Medical Center

State University of New York

Brooklyn, New York 